\title{
Fascistissimi sempre. II fascismo nella letteratura italiana
}

Il saggio individua alcune costanti nella rappresentazione del fascismo offertaci da scrittori italiani di vario orientamento in special modo fra anni Trenta e Settanta del Novecento. Ad accomunare la maggioranza dei testi vagliati risulta essere l'idea che il fascismo sia stato e, in forme sempre nuove, possa ciclicamente tornare ad essere il destino quasi naturale di un'Italia incline alla deriva autoritaria, perché storicamente governata da una borghesia tanto retriva quanto dispotica.

This essay identifies a series of common elements in the representation of Fascism provided by Italian authors of different orientations, especially between the Thirties and Seventies of the Twentieth century. The main idea found in all of the examined texts is that Fascism was, and somehow has the potential to cyclically become again, the natural destiny of Italy, considering the inclination toward authoritarianism shown by a Country that has traditionally been governed by a conservative and despotic middle class.

\section{Buco nero}

Premessa inevitabile: senza nessuna pretesa di stilare 1'elenco esaustivo delle opere letterarie che hanno trattato il fascismo, e limitandosi peraltro a sondare alcuni dei testi in prosa in tale ottica più fecondi, si cercherà solo di rendere tra loro congrui spunti interpretativi magari utili a una futura disamina, essa sì rigorosa, delle tendenze che hanno guidato - sia in presa diretta, sia a posteriori - le differenti ricognizioni almeno narrative del Ventennio. E che le pagine seguenti si pensino quindi alla stregua di un provvisorio carotaggio esegetico regala al loro 
autore un di più d'impertinenza critica. Ad esempio, quella di anticipare allusivamente le parziali acquisizioni di senso ricavate da siffatta rassegna ermeneutica rischiando subito una provocazione: e se il più acuto ritratto del duce restasse quello propostoci, in un racconto del 1929, Mario e il mago, non da uno scrittore italiano, ma da Thomas Mann? Perché l'impressione è che veda giusto Raffaeli: molto «si è detto dello stato di minorità secolare della prosa italiana e massime della forma-romanzo surrogata dalla prosa d'arte, che infatti è il genere più filisteo e più culturalmente accreditato sia della evasione sia della ornata mistificazione»; ma poco si è «analizzata la difficoltà ad elaborare lo spazio-tempo dove culmina la cosiddetta autobiografia italiana (e dunque il fascismo) per il quale è passata, serbandone tracce evidenti, la generazione di scrittori che ha incarnato, alla lettera, il Novecento italiano» [Raffaeli 2014, vii].

La nostra narrativa non sembra cioè conoscere indubbi capolavori che stiano al desiderio di elaborare, magari trasfigurandole, un'archeologia e, al tempo stesso, una decodifica intellettuale del fascismo come il Doctor Faustus (1947) del succitato Mann o la trilogia I sonnambuli (1931-1932) e, soprattutto, Gli incolpevoli. Romanzo in undici racconti (1950) di Broch stanno all'urgenza di cogliere i fondamenti storico-antropologici del nazismo traslitterandoli in monumentali operemondo. A parte le eccezioni cui ci si riferirà, annovera, questo sì, vari tentativi, per così dire, più epidermici di restituire ai lettori istantanee, resoconti o disamine dell'epoca mussoliniana a basso tasso di reinvenzione allegorico-romanzesca. Sforzi che tuttavia rischiano spesso di scivolare o nell'affresco bozzettistico, nell'ossessione cronachistica, nell'ovvio zelo didascalico tipici, per l'appunto, della nostra prosa d'arte o in una memorialistica sovente incline all'aneddotica anche perché in genere troppo letterariamente compiaciuta, solo di rado nutrita da una coerente teoria critica.

Per un verso, basti considerare il Pratolini già di Un eroe del nostro tempo (1949), poi dei due romanzi, Lo scialo (1960) e Allegoria e derisione (1966), che - l'uno descrivendoci l'avvento del fascismo e il processo di involuzione morale di quella borghesia organica al regime, l'altro rivisitando gli anni del secondo conflitto mondiale e la Resistenza - formano insieme a Metello (1955) - rievocazione delle lotte operaie tra Otto e Novecento - la trilogia Una storia italiana. Dall'altra parte, viene invece in mente Bilenchi. Non tanto per Il bottone di Stalingrado (1972), testo che narra «in tre tappe la formazione del protagonista Marco: fascismo; resistenza e liberazione; dopoguerra, conflitti sociali e repressioni antioperaie» [Centovalli 2009, xxii]. Piuttosto, per quel sia pur prezioso «romanzo in progress» deliberatamente in bilico tra autobiografia intellettuale, ritrattistica storico-politica e racconto che è Amici (1976), capace, saldandosi al suo ideale 
secondo atto, il postumo Due ucraini e altri amici (1990), di realizzare il proposito dell'autore: stendere, alla propria maniera, «il romanzo della generazione» cui egli apparteneva e «che aveva creduto negli ideali di un fascismo operaio e rivoluzionario e aveva fatto la guerra e la Resistenza nelle file del pci clandestino» [Centovalli 2009, xx-i].

Ad ogni modo - e lo si rimarca non a disdoro dei primi, ma a merito dei secondi-, se poi si esaminano, al di là dei giudizi appena formulati, le analisi del Ventennio complessivamente offerteci dai principali prosatori italiani, ci si accorge che esse collimano - quando per consapevole riuso, quando per autonome deduzioni - con le chiavi di lettura proposteci da alcuni di quegli intellettuali cui siamo tradizionalmente usi affidarci per indagare tale stagione della nostra storia recente. Anche per la maggior parte degli scrittori preoccupati di intenderne genesi, essenza, lasciti, il fascismo non rappresenta, ad esempio, un incidente di percorso per l'identità italiana, ma il suo massimo disvelamento: a volerla dire con la già citata formula di Gobetti, il capitolo cruciale dell' «autobiografia della nazione» [Gobetti $2008,165]$. E quella che trova nel corpo sempre in posa del duce il suo perno non solo simbolico è di fatto descritta da molti romanzi o racconti - pur senza magari ricorrere a simili categorie critiche messe a punto o rivisitate da Gramsci - come una dittatura di classe, quindi borghese, da pensarsi quale ennesima «rivoluzione passiva» subita da un Paese che, schiavo del «trasformismo», pare ciclicamente costretto a conoscere stagioni di mutamenti socioculturali anche profondi che però non si rivelano mai il risultato di autentiche spinte progressive, ma si confermano ogni volta l'esito del perverso abbraccio tra un cinico «sovversivismo» dall'alto e un qualunquistico «sovversivismo» dal basso, giacché la nazione solamente vede, a ispirare e gestire tali processi di fittizia modernizzazione, quei gruppi egemoni capaci, grazie ai falsi rinnovamenti imposti, di favorire l'adeguamento degli stili di vita e delle logiche di giudizio delle masse ai propri interessi specifici, al rifiuto di qualsivoglia «dominio della legge», alla legittimazione di una politica «di arbitrii e di cricca» [Gramsci 1996a, 85-92; Gramsci 1996b, 86-8, 118, 171, 196-8; Gramsci 1996c, 17-20]. Così, che sia insomma incline a credere il fascismo la più turpe epifania di quel «trasformismo» tipicamente italiano che ha per scopo «il potere come tale» e va quindi considerato «violenza mascherata» sempre pronta a cedere il passo alla «violenza aperta» [Bollati 2011, xxx, xxxii], anche implica che molta nostra narrativa scorga nel regime il sintomo storicamente più grave $\mathrm{e}$ la conferma in assoluto peggiore di una malattia etica $\mathrm{e}$ un ritardo culturale parimenti colpevoli - segnando fin dal principio la borghesia nazionale - di determinare la cronica cattiva salute del Paese. 


\section{Estremisti a corte}

Da un lato, questa interpretazione del Ventennio come metastasi dell'incurabile tumore borghese che debilita l'Italia trova un riscontro nella parabola dei cosiddetti "fascisti di sinistra": tra i tanti, Vittorini o il già citato Bilenchi. Per esempio, i pamphlet licenziati da quest'ultimo nei primi anni Trenta, Vita di Pisto (1931) e Cronaca dell'Italia meschina ovvero Storia dei socialisti di Colle (1933), svelano che costoro furono indotti dal proprio «estremismo giovanile» ad approvare lo «squadrismo delle origini» perché vi rintracciarono, erroneamente, quella «rivolta antiborghese» da loro auspicata, quindi un'escrescenza dell'«anticapitalismo romantico» da cui erano animati, e che essi divennero oppositori del regime appena vi riconobbero una dittatura, per l'appunto, disposta ad assicurare «la glaciale conservazione dell'ordine costituito», ossia del «capitalismo all'italiana, retrivo e bigotto, antipopolare e antioperaio per antonomasia» [Raffaeli 2014, 6-9]. E anche senza voler considerare i suoi migliori racconti satirici o saggi di costume - Il vecchio con gli stivali (1945), I fascisti invecchiano (1946) -, gli stessi romanzi concepiti da Brancati dopo l'allontanamento dal fascismo e il ripudio del giovanile vitalismo di marca dannunziana - Gli anni perduti e Don Giovanni in Sicilia (1941), Il bell'Antonio (1949), l'incompiuto Paolo il caldo (apparso postumo nel 1955) -, dapprima con barocca, tragicomica vena sarcastica e, infine, con cupo furore allucinatorio, individuano nel "gallismo", che logora gli inetti maschi catanesi, il ferale aggravamento della patologia borghese considerata intrinseca all'imperio mussoliniano.

Persino nelle diagnosi di alcuni scrittori rimasti sempre fedeli al regime si osserva l'errore di valutazione compiuto in gioventù dai fascisti poi pentitisi. Col libro in cui rievoca la sua esperienza di combattente volontario in Africa tra il $1942 \mathrm{e}$ il 1943, Guerra in camicia nera (1955), Berto, lungi dall'offrirci - come s'era provato a fare Flaiano in Tempo di uccidere (1947) ironizzando sulla campagna di Etiopia del 1936 alla quale aveva preso parte - la surreale, allegorica dissacrazione di ogni retorica patriottico-militarista e, di riflesso, dell'ideologia incarnata dal duce, ci ricorda che, all'epoca, molti italiani, al pari suo, continuavano a credere che il fondamento della dittatura fosse quel desiderio di «"andare verso il popolo"» da realizzarsi, secondo loro, arrivando anche a ipotizzare «la necessità» di una «rivoluzione postbellica» concepita alla stregua di «una rivoluzione nel fascismo e non contro il fascismo» [Berto 2010, 39-40]. Lo stesso Berto catturato dagli alleati nel 1943 e condotto negli Stati Uniti, qui trasferito in vari campi di concentramento - fino a giungere a Hereford, come attesta Prigionieri nel Texas (1985), in cui Tumiati narra la detenzione di alcuni nostri connazionali in tale 
struttura carceraria - e che poi, tornato libero in Italia, pubblica tre romanzi di ispirazione neorealista ambientati durante la seconda guerra mondiale: Il cielo è rosso (1947), Le opere di Dio (1948), Il brigante (1951).

Vicenda - quella anzitutto dei fascisti di sinistra ma, specularmente, anche degli scrittori mai rivelatisi ostili al duce, benché poi solleciti nel presentare al pubblico come da sempre loro i valori egalitari della neonata repubblica - che suggerisce due rapide osservazioni. Intanto, che forse i malumori dei primi, pur non ammessi, furono sì puniti, ma principalmente sorvegliati da un regime che, a ben guardare, neppure esagerò quanto si potrebbe magari credere nel celebrare gli entusiasmi, certo graditi, dei secondi, perché Mussolini, in quella sua politica propagandistica orientata a costruire l'immaginario dei connazionali, pare scommettere molto di più su altre forme d'arte o di comunicazione - il cinema, la radio - che non sulla letteratura, quasi egli già la consideri ciò che da tempo essa è realmente divenuta - un discorso socialmente marginale, se non estinto - o voglia comunque evitare di ripetere l'esperimento tentato da un suo maestro di teatralità: quel D'Annunzio che aveva fatto leva precisamente sull'estetizzazione del linguaggio poetico, cioè sulla spettacolarizzazione della propria aura di vate, per «mettere a punto la figura di un demiurgo capace di infiammare e di dirigere in presa diretta le masse» [Bollati 2011, xxxii]. E poi che, qualsivoglia momento della storia patria si prenda in esame, gli intellettuali italiani sembrano invariabilmente oscillare tra un massimalismo (in verità, soprattutto di parole) e un servilismo (sempre negato, eppure reso generalmente inconfutabile dai fatti) che tendono, però, non a radicalizzare il loro antagonismo, ma a rovesciarsi l'uno nell'altro, così generando le ricorrenti, complementari figure dell'estremista di corte e del contestatore per conformismo.

\section{II belluino senso dell'orrore}

Dall'altro lato, che la letteratura italiana ritragga perlopiù il fascismo come un gattopardesco gioco di prestigio della nostra geneticamente lobbistica classe egemone è un'evidenza da cui possiamo ricavare un valido criterio per decifrarne gli esiti in tale ottica migliori. Senza dubbio, Il romanzo di Ferrara di Bassani, opera in progress, apparsa in edizione definitiva nel 1984, che include testi concepiti a partire dal 1938 (i cicli di racconti Dentro le mura e L'odore del fieno; i romanzi Gli occhiali d'oro, Il giardino dei Finzi-Contini, Dietro la porta, L'airone), via via pubblicati come volumi autonomi e che sembrano prefiggersi lo stesso obiettivo degli Incolpevoli: dimostrare che, all'origine del fascismo, vi fu, incarnato da Mussolini, quello «spirito piccolo borghese» (tipico «dell' animale rapace prude», pronto ad ammettere «qualsiasi atrocità») che, trovata in Hitler la propria per- 
sonificazione, Broch giudica, in una Nota sulla genesi del romanzo, l'autentica matrice del nazismo [Baldacci 1970, 11]. E poi, naturalmente, Eros e Priapo (Da furore a cenere), il pamphlet del 1967 in cui Gadda sostiene che «tutta la ventennale soperchieria è contraddistinta dai caratteri estremi della scempietà, della criminalità puerile, della mancanza di senso e di cultura storica: non diciamo del senso etico e religioso» [Gadda 1992, 244].

Come pure consente, la tesi di fondo sul regime quale cancerogeno tessuto borghese ribadita da tanta nostra narrativa, di rinvenire in Moravia l'autore che, di tale diagnosi, ha saputo proporci forse per primo una compiuta, e in seguito troppo pigramente reiterata, formalizzazione romanzesca. Ci si sta ovviamente riferendo agli Indifferenti (1929), «documento di quasi saggistica lucidità» scaturito «da un diretto desiderio di analisi, precisamente, moralistica e satirica, della società borghese, nello stadio storico della sua dissoluzione decadente, e segnatamente negli anni del fascismo italiano» [Sanguineti 1977, 9]. E poi non tanto alla Mascherata (1941), apologo satirico i cui idoli polemici sono certamente i regimi nazifascisti, e in fondo neppure alla Romana (1947) o alla Ciociara (1957), bensì al Conformista (1951). Libro impeccabile per una cinquantina di pagine, ossia in quel Prologo in cui Moravia precisa che l'attitudine all'omologazione connaturata alle nostre élites dominanti, oltre a rivelarsi l'altra faccia del loro "nichilismo passivo", già esplorato nel volume d'esordio, appare intrinsecamente squadrista, e non poteva quindi che generare il fascismo, in quanto rovescia un «gusto della distruzione e della morte» in «felicità aggressiva e crudele», un costante terrore di scoprirsi «anormali» nel desiderio di uniformarsi a «un ordine purchessia» [Moravia, 2015, 54, 60-1]. Nei capitoli successivi, testo che cade però vittima del suo «artificioso traliccio narrativo», risultando «uno dei romanzi più infelici e macchinosi» dello scrittore [Sanguineti 1977, 64]. Nonché un'opera che aspira parimenti ad esprimere «un paradosso maligno», cioè raffigurare - come ammise lo stesso Moravia - «l'incapacità borghese di un radicale antifascismo», ma che sceglie di farlo attraverso «una caricatura ignobile» di Carlo Rosselli (cugino dell'autore) e della moglie Marion, ritratti «nelle vesti di personaggi grotteschi e velleitari» [Ventura 2017, 190]. Quasi a voler confermare che Moravia «certo non aveva alcuna simpatia per il fascismo, ma neppure per l'antifascismo», giacché «guardava alla politica col distacco del letterato, senza impegnarsi» [Ventura 2017, 190]. 


\section{Perdute lotte di classe}

Sbirciamo allora nella prosa italiana e proviamo a vagliare alcuni titoli apparsi tra la pubblicazione degli Indifferenti e quella del Conformista. Ci accorgiamo subito che, pur magari nascendo da orientamenti culturali diversi, i migliori tentativi di raccontare il Ventennio tendono però tutti a canonizzare l'esegesi del regime, quale camaleontica convalida della sovranità borghese, fin qui riassunta.

Esemplare, in tal senso, Marcia su Roma e dintorni (1931), perché Lussu sia ci regala «una interpretazione già compiutamente storiografica» del fascismo come occorrenza della «eterna vicenda del trasformismo italiano», sia «rifiuta politicamente il vecchio antifascismo degli sconfitti (impietosa è la sua descrizione dell'Aventino)» per avventurarsi «coraggiosamente alla ricerca di un nuovo antifascismo, da far nascere obbligatoriamente sulle macerie lasciate dai compromessi giolittiani, dall'impotenza dei riformisti, dal velleitarismo dei massimalisti» [De Luna 2014, 4-6]. Strada a quel tempo quasi deserta, come sembrano da altra prospettiva dichiarare anche i saggi - Il fascismo. Origini e sviluppo (1934), La scuola dei dittatori (1938) - e i romanzi - Fontamara (1933), Vino e Pane (1937), Il seme sotto la neve (1941) - di Silone, che, nelle sue prove narrative, mostra parimenti di considerare il Ventennio l'approdo di una storia, la nostra, invariabilmente fondata, specie nel Meridione, sugli abusi di potere dei "padroni" nei confronti di quei "cafoni" spesso spinti dalla disperazione a tramutarsi nei sicari dei propri aguzzini. Diagnosi simile a quella che, individuando la causa della sostanza perennemente dispotica della società italiana nell'esito giocoforza scontato dell'impari lotta tra "Luigini”" e "Contadini”, Carlo Levi formulerà, dopo avercene dato un'anticipazione in Cristo si è fermato a Eboli (1945), in uno dei massimi libri civili del nostro Novecento: L'Orologio (1950). Pubblicato l'anno stesso in cui, postuma, appare un'altra opera ascrivibile all'identica linea esegetica: Le terre del Sacramento di Jovine.

E L'Orologio - definendo la guerra partigiana la prima, vera "rivoluzione contadina" esperita dal Paese, ma altresì una vittoria di cui i Luigini cercano subito di appropriarsi per ribadire il loro diritto al comando nella nascente repubblica - tenta anche, a ridosso di analoghi sforzi per esempio compiuti da Vittorini e Pavese, un precoce e tuttavia già lucido bilancio politico della Resistenza parimenti legittimato da quelle valutazioni storico-antropologiche dell'identità italiana che avevano appunto suggerito a molti di scorgere nel cesarismo di Mussolini uno sbocco purtroppo logico per la nazione. Dopo aver pubblicato in volume quella allegoria antifascista che è Conversazione in Sicilia (1941) e prima di fare la stessa cosa con Il garofano rosso (1948) - che egli presenterà alla stregua di un 
documento sul «granchio» preso al principio dalla sua generazione, disposta, in gioventù, ad aderire al fascismo credendolo «rivoluzionario» e dunque dal sicuro «contenuto socialista» [Vittorini 1997, 191-2] - Vittorini aveva infatti licenziato Uomini e no (1945). Che Pavone riterrà, com'è noto, un «brutto romanzo di argomento resistenziale», colpevole, fin dal titolo, di fare propria - preciserà lo studioso citando un giudizio di Franco Calamandrei - un'astorica formula «esorcistica» esclusivamente preoccupata di rintracciare, nella lotta tra partigiani e repubblichini, non una «guerra civile», di cui allora sorvegliare attentamente l'esito politico nella successiva età repubblicana, ma un'astratta, quasi metafisica contesa tra individui eticamente degni e squallide belve sanguinarie [Pavone 2006, 222]. Laddove provvederà proprio Pavese a raffigurare lo scontro tra fascisti $\mathrm{e}$ antifascisti appunto come una guerra civile: in maniera esplicita nella Casa in collina (1948); per via indiretta nel Compagno (1947), nel Carcere (1948), nella Luna e i falò (1950).

Tutto ciò mentre Savinio raccoglie in Sorte dell'Europa (1945) vari articoli, editi tra il 25 luglio del 1943 e la fine dell'anno successivo, in cui esorta a riconvertire la vittoriosa lotta di liberazione dal nazifascismo in un disegno politico orientato a fare del Vecchio Continente «una sola nazione unita da comuni pensieri, da comuni interessi, da un comune destino» [Savinio 1977, 84]. Mentre Malaparte - in passato autore di un'opera narrativa, Don Camaleo. Romanzo di un camaleonte (1927), che accusava di tirannico trasformismo Mussolini, nonché di un trattato, Tecnica del colpo di Stato (1931), da cui si evinceva un'interpretazione del fascismo non quale moto rivoluzionario ma, appunto, come golpe - affida a Kaputt (1944) e alla Pelle (1949) i suoi timori sul rischio che l'ecatombe causata dalla seconda guerra mondiale diventi, per l'Europa, un trauma che essa, condannandosi quindi a vivere in una sorta di coma vigile, mai riuscirà a superare. E, soprattutto, mentre ad imporsi come pur implicita rappresentazione compiuta del regime, e anzi - in anticipo «sull'avveniristico 1984 di Orwell e sulla testimonianza di Buio a mezzogiorno di Koestler» - quale convalida di quella «minaccia di un totalitarismo globale» che spaventa ancora molti [Borsellino 2006, 11], è un libro di Alvaro, autore che in verità, firmando Terra nuova. Prima cronaca dell'Agro Pontino (1934), era parso a suo tempo schierarsi con il regime. Si allude, naturalmente, all'Uomo è forte (1938), affresco sulla «condizione dell'uomo in uno stato di terrore» [Alvaro 2006, 21] scaturito dalla rielaborazione romanzesca di quelle corrispondenze dalla patria della rivoluzione d'ottobre, visitata nel 1934, che lo scrittore aveva raccolto nei Maestri del diluvio. Viaggio nella Russia sovietica (1935). 


\section{Autoritaria repubblica borghese}

Conseguenza del ragionamento fin qui svolto è che non ci si debba dunque stupire se, via via che ci si allontana dal dopoguerra e sino a tutti gli anni Settanta almeno, i principali testi letterari inclini a rievocare il Ventennio convalidano l'ormai classica analisi del regime come epifania tanto della ineducabile natura dispotica della nostra borghesia, quanto della matrice in sé reazionaria dell'identità italiana, per riferirsi al costante rischio della deriva autoritaria in una repubblica sia pur figlia della Resistenza o, addirittura, per dichiarare strutturalmente liberticida, quindi cripto-fascista, la nostra democrazia. È ciò che fanno Lino Dal Fra, Cecilia Mangini e Lino Miccichè in All'armi siam fascisti! (1962), documentario di montaggio che affida a un commento scritto da Fortini il compito di unificare i materiali di repertorio, perlopiù inediti, proposti al pubblico. E sono queste anche le intenzioni, più o meno scoperte, di molti testi, variamente classificabili, che si confrontano, quando tangenzialmente o in maniera solo allusiva e quando di petto, con l'urgenza di sondare l'eredità lasciata al Paese dal fascismo.

Le intenzioni, per esempio, già rinvenibili in quella memoria autobiografica sul proprio apprendistato di scrittore in epoca mussoliniana che è l'Introduzione (1956) di Delfini alla ristampa accresciuta di una sua raccolta di racconti, Il ricordo della Basca (1938), e però anzitutto rintracciabili in quei Fiori italiani (1976) in cui Meneghello - regalandoci l'anello di congiunzione tra Libera nos a malo (1963) e Pomo pero (1974), lacerti essi pure di quell'autoritratto di un italiano che, con I piccoli maestri (1964), include anche la rivisitazione della lotta partigiana - ci consegna «sapide pagine» su un insegnamento scolastico che ai tempi del duce «non era nemmeno indottrinamento fascista specifico e diretto», perché appariva invece l'esito della cronica fedeltà a «una pesantemente esclusivista tradizione di educazione miopemente classista, reazionaria nell'intrinseco, fascista ma più antica del fascismo», mantenutasi «a lungo nelle pieghe della scuola nell'età della repubblica» [De Mauro 2007, v-vi]. E le intenzioni ugualmente sottese, come in Memorie dell 'incoscienza (1954) di Ottieri, a stupefatte denunce dell'ipocrisia di una nazione entusiasta di Mussolini ma, caduto il regime, d'un tratto scopertasi antifascista da sempre. A dissacranti analisi delle logiche sociali che ispirano la ricostruzione postbellica o i processi di industrializzazione quali Ragazzi di vita (1955) di Pasolini e Tempi stretti (1964) ancora di Ottieri. A pur tra loro imparagonabili romanzi di formazione, ambientati nel Ventennio o durante la seconda guerra mondiale, come Il ragazzo morto e le comete (1951) e Il prete bello (1954) di Parise, Il lanciatore di giavellotto (1981) di Volponi. E, in fondo, 
anche ad Oro, il racconto, incluso nel Sistema periodico (1975), in cui Primo Levi descrive la sua cattura da parte dei fascisti dopo una breve esperienza partigiana. E se, in molte opere letterarie del secondo Novecento italiano, a serpeggiare è insomma la tesi che - per dirla col titolo inizialmente previsto da Volponi per un romanzo, La strada per Roma (1991), ideato sul finire degli anni Cinquanta ma concluso oltre un trentennio più tardi - la nostra sia una Repubblica borghese, $\mathrm{e}$ dunque immutabilmente classista, neppure ci si può meravigliare del fatto che una simile tendenza ad interrogarsi sul fascismo anzitutto per riflettere sulla reale portata delle successive conquiste democratiche della nazione si esasperi - a prescindere dai giudizi dei singoli scrittori sulle diverse rivolte giovanili o sulle varie lotte operaie - durante le pur distinte fasi di quella contestazione che, accusandolo per l'appunto di conservare una struttura autoritaria, scuote il Paese negli anni Sessanta e Settanta. La Storia (1974) di Elsa Morante, ad esempio, va letto anche in quest'ottica.

Ecco perché, a tale altezza, la rappresentazione letteraria del fascismo, da sempre propensa a saldarsi con la rivisitazione delle esperienze resistenziali, mostra di poter ormai duplicare la parabola tracciata da quest'ultima, in egual misura disposta - dopo aver salutato esiti già felici nel Sentiero dei nidi di ragno (1947) e in alcuni racconti confluiti in Ultimo viene il corvo (1949) di Calvino; essersi lasciata alle spalle intimistici tentativi di sminuire o squalificare gli orientamenti politici delle lotte partigiane come quelli compiuti dal Cassola di Fausto e Anna (1952), I vecchi compagni (1953), La ragazza di Bube (1960); aver trovato le proprie perle grazie a Fenoglio, quindi nei Ventitré giorni della città di Alba (1952), in Una questione privata (1963), nel Partigiano Johnny (1968) - a farsi modulare dagli autori, o percepire comunque dal pubblico, alla stregua di un'implicita esortazione, rivolta ai lettori del tempo, a ribellarsi a un ordine costituito supposto liberticida. Benché non narri le lotte partigiane, il radiodramma Parma 1922. Una resistenza antifascista (1973) manifesta appunto un'intenzione simile, perché Balestrini ricostruisce le cinque giornate dell'agosto 1922 che videro i rioni popolari della città emiliana opporsi in armi alle incursioni delle camicie nere di Balbo. Di riflesso, in sintonia con gli umori di una certa sinistra, il tacito assunto diviene all'incirca questo: contro la vigente civiltà criptofascista urge di nuovo scommettere su una resistenza che però, a differenza di quella storica, va trasformata in una rivoluzione in nome dell'equità sociale, evitando così di "tradire" essa pure.

Ma è il letterato maggiormente critico con il movimento studentesco del '68, che in sostanza considera l'ennesima "rivoluzione passiva" inscenata dalla nostra borghesia, nonché l'intellettuale più spregiudicato nell'addebitare a quest'ultima 
la responsabilità di aver ridotto l'Italia repubblicana a un'edonistica società totalitaria, da cui non crede possibile reperire via di fuga alcuna: è insomma Pasolini l'autore che, tra anni Sessanta e Settanta, più di ogni altro converte qualsiasi germe di ragionamento sulla dittatura mussoliniana in una riflessione metastorica sul regime volta a denunciare l'emersione, nel presente, di una forma nuova, persino peggiore, di fascismo. È la tesi che ispira l'intera produzione ultima dello scrittore e cineasta. Che emerge, per esempio, dalle sue tragedie in versi-Orgia, Bestia da stile, Pilade, Affabulazione, Porcile, Calderón - stese tra il 1966 e il 1967 ma, specie alcune, a lungo ripensate. Che guida quel rifacimento della raccolta di poesie La meglio gioventù (1954) licenziata col titolo La nuova gioventù (1975). Che si ripete ossessivamente nei saggi confluiti negli Scritti corsari (1975) e nelle Lettere luterane (1976). Che determina la forma di Petrolio (1992), il romanzo incompiuto cui Pasolini lavora a partire dal 1972, dunque negli ultimi tre anni di vita.

\section{Forward, rewind}

Pur smorzando decisamente i toni, la letteratura italiana ha fatto propria la decodifica del fascismo come "autobiografia della nazione" anche in seguito: vengono alla mente alcune pagine dell'Oro del mondo (1987) e di Cuore di pietra (1996) di Vassalli o del recente La gemella $H$ (2014) di Falco. Ed essa ha a maggior ragione riproposto tale chiave di lettura quando ha inteso decifrare il berlusconismo, magari interpretabile come un regime, solo in apparenza non autoritario, scaturito, al pari di quello mussoliniano, dal congenito "sovversivismo" della nostra classe dominante. Non per nulla, Balestrini intitola Una mattina ci siam svegliati (1995) il testo polifonico in cui celebra la grande manifestazione nazionale del 25 aprile svoltasi un mese dopo il primo successo elettorale riscosso da Berlusconi. Tutt'altro sono invece i due volumi di cui consta Canale Mussolini (2010; 2015) di Pennacchi: un tentativo di costruire il grande romanzo epico italiano in larga parte fallito. Magari anche perché abbozzato da un'ulteriore incarnazione di quella maschera di estremistico intellettuale qualunquista ricorrente nella nostra storia patria. 


\section{Bibliografia}

Alvaro C. 2006, L'uomo è forte, Soveria Mannelli: Rubbettino

Baldacci L. 1970, Introduzione, in Bassani G. 1970, Gli occhiali d'oro, Milano: Mondadori

Berto G. 2010, Guerra in camicia nera, Milano: Rizzoli

Bollati G. 2011, L'Italiano. Il carattere nazionale come storia e come invenzione, Torino: Einaudi

Borsellino N. 2006, Prefazione, in Alvaro 2006

Centovalli B. 2009, Quattro ragioni più una per leggere Bilenchi, in Bilenchi R. 2009, Opere complete, Milano: Rizzoli

De Luna G. 2014, Introduzione, in Lussu E. 2014, Marcia su Roma e dintorni, Torino: Einaudi

De Mauro T. 2007, Introduzione, in Meneghello L. 2007, Fiori italiani con un mazzo di nuovi Fiori, Milano: Rizzoli

Gadda C.E. 1992, Saggi Giornali Favole e altri scritti, Milano: Garzanti

Gobetti P. 2008, La Rivoluzione Liberale. Saggio sulla lotta politica in Italia, Torino: Einaudi

Gramsci A. 1996a, Note sul Machiavelli, sulla politica e sullo Stato moderno, Roma: Editori Riuniti

Gramsci A. 1996b, Il Risorgimento, Roma: Editori Riuniti

Gramsci A. 1996c, Passato e presente, Roma: Editori Riuniti

Moravia A. 2015, Il conformista, Milano: Bompiani

Pavone C. 2006, Una guerra civile. Saggio storico sulla moralità nella Resistenza, Torino: Bollati Boringhieri

Raffaeli M. 2014, I fascisti di sinistra e altri scritti sulla prosa, Torino: Aragno

Sanguineti E. 1977, Alberto Moravia, Milano: Mursia

Savinio A. 1977, Sorte dell'Europa, Milano: Adelphi

Ventura A. 2017, Intellettuali. Cultura e politica tra fascismo e antifascismo, Roma: Donzelli

Vittorini E. 1997, Il garofano rosso, Milano: Mondadori

\section{Risorse online}

Video della relazione al convegno Narrare il fascismo (Predappio, 20-21 gennaio 2017) https://www.youtube.com/watch?v=uJC1r8O-Ldg 